\title{
DER GEMEINSAME ROHSTOFF-FONDS EIN MARKTWIRTSCHAFTLICHES INSTRUMENT?
}

\author{
VON JOACHIM BETZ
}

\section{Einleitende Betrachtungen}

In der Bundesrepublik kann - zumindest was die von der Bundesregierung vertretene Position und die Schlußfolgerungen wenigstens einiger wirtschaftswissenschaftlicher Institute anbetrifft - von einem erheblichen Meinungswandel hinsichtlich des von der Gruppe der 77 geforderten Gemeinsamen Rohstoffonds gesprochen werden, der ja eines der Kernbestandteile der Neuen Weltwirtschaftsordnung darstellen soll. Der Gemeinsame Fonds, so verlautete von der Bundesregierung anfänglich, stelle die arbeitsteilige, auf dem Prinzip der Handelsfreiheit beruhende Wirtschaftsordnung in Frage und ersetze sie durch ein System globaler Reglementierungen (D. Genscher) ${ }^{\mathbf{1}}$. Das Programm verschlinge ,, ungeheure Geldmittel", heize die Inflation an und verbaue den danach verlangenden Entwicklungsländern selbst den Weg zu solidem Wohlstand durch eine Fixierung der Produktionsstrukturen auf den Rohstoffsektor (H. Friderichs) ${ }^{2}$. Monopolistische Praktiken, Rohstoffdirigismus und mechanistische Systeme seien kein Weg, um die Wohlstandslücke zwischen armen und reichen Ländern zu schließen $(\mathrm{H} \text {. Schmidt })^{3}$, am Ende dieses Weges stünden vielmehr ,,monströse globale Bürokratien, weltweiter Immobilismus und Ressourcenvergeudung" (M. Schlei) ${ }^{4}$. Später wurde dann auch vor allem auf die gefährliche Nähe dieses Konzeptes zum EG-Agrarmarkt hingewiesen, da bei manipulierten und zu hoch angesetzten Preisen die daraus resultierende Úberproduktion vom Markt genommen werden müßte, mit der Folge riesiger und teuer zu lagernder Rohstoffberge $(\mathrm{H} \text {. Friderichs })^{5}$. Zur Abwehr dieser Folgeprobleme seien weitere dirigistische Eingriffe in den Welthandel (Exportquoten, Abnahmeverpflichtungen) unausweichlich. Nicht zuletzt würden zu den Gewinnern des Rohstoffprogramms auch einige Industrieländer zählen, während eine erhebliche Anzahl rohstoffarmer Entwicklungsländer auf der Verliererseite stünde.

Einige Argumente wurden von der Bundesregierung (und natürlich in noch verschärfter Form von der CDU/CSU und den Wirtschaftsverbänden) bis März 1977 nahezu ungeschmälert vertreten, wenn auch im Laufe der praktischen Verhandlungen (UNCTAD, KIWZ, EG-Gipfel) zunehmend Konzessionen gemacht wurden, die von der Zustimmung zu Rohstoffabkommen im Einzelfall zur Zustimmung zu einem Common Fund (im folgenden $=\mathrm{CF}$ ) in der Form eines Ressourcenpools führten. Lediglich das BMZ konnte sich schon ab Ende Oktober 1975 auch theoretisch mögliche Vorteile aus einem Entgegenkommen im Rohstoffbereich versprechen, nämlich Versorgungssicherheit mit Rohstoffen zu angemessenen Preisen, die Beseitigung von Ungewißheiten im Verhältnis zu den Entwicklungsländern und Sicherheit für Investoren im Rohstoffbereich ${ }^{6}$. Damit stand das BMZ freilich lange Zeit allein.

\footnotetext{
1 Die Welt v. 24. 9. 74, Genscher: Zum Frieden gehört das Recht auf Selbstbestimmung.

2 Die Welt v. 29. 4. 1975, Ohne Freiheit kein Ja zur Neuordnung.

3 FAZ v. 4. 10. 75, Schmidt: Industrieländer müssen besser zusammenarbeiten.

4 M. Schlei, Sozialer Ausgleich im Weltmaßstab, in: Die Neue Gesellschaft, Mai 1977.

5 Rheinischer Merkur v. 10. 12. 76, Nord-Süd-Dialog.

6 Vgl. Handelsblatt v. 27. 10. 75, Kompromißformeln für die Entwicklungspolitik.
} 
Im März 1977 plädierte der Vorsitzende des BT-Ausschusses für wirtschaftliche Zusammenarbeit, Uwe Holtz, mit Nachdruck für die Unterstützung eines Gemeinsamen Fonds, freilich in noch reduzierterer Form, als er später von der Gruppe B (den westlichen Industrieländern) als Vorschlag eingebracht wurde 7 . In den Leitlinien der SPD zur Nord-Süd-Politik (Oktober 1977) heißt es dann bereits: ,,Das integrierte Rohstoffprogramm enthält berechtigte Forderungen der Entwicklungsländer. Es läßt sich marktgerecht ausgestalten. Dies gilt insbesondere für Rohstoffabkommen und den Gemeinsamen Fonds ${ }^{8}$ " Zur selben Zeit äußerte sich auch bereits Bundesaußenminister Genscher ähnlich positiv zu preisstabilisierenden Rohstoffabkommen, denen er erhebliche Vorteile für Produzenten (geordnete Durchführung der Entwicklungspläne, Reduktion des Investitionsrisikos) wie auch für Konsumenten(stabile Preise, Versorgungssicherheit, Verstetigung von Konjunktur und Beschäftigung) zuschrieb: ,, Rohstoffabkommen sind nicht eo ipso dirigistisch und marktzerstörend. Es kommt vielmehr auf die Ziele und die Ausgestaltung des Abkommens an9."Man wird sich fragen, wie ein Programm, das eigentlich zur Suspension des Marktes führen sollte, binnen kurzem zu einem durchaus marktkonformen und nützlichen Vorhaben avancieren kann. Die Antworten hierauf könnten so lauten:

1. Die Forderungen der Gruppe der 77 haben sich in Richtung marktkonformer Vorstellungen verändert.

2. Der Gemeinsame Fonds war von seiner Konzeption her nie antimarktwirtschaftlich ausgelegt. Gegenteilige Äußerungen der Bundesregierung beruhen auf Unkenntnis bzw. falscher Folgeneinschätzung oder aber auf mehr oder minder bewußter Panikmache.

3. Der Gemeinsame Fonds wird von der Regierung heute nicht anders wie damals beurteilt, mangels akzeptabler oder glaubwürdiger Alternativen bleibt jedoch - besonders auch hinsichtlich der außenpolitischen Schadensbegrenzung - nur ein Eingehen auf die Vorstellungen der ,, $77^{\prime \prime}$ in der Hoffnung, während der Verhandlungen noch hier und dort marktwirtschaftskonforme Besserungen einfügen zu können.

Die erste These kann ohne weiteres verworfen werden: Die Vorstellungen der , 77 “ haben sich zumindest zum CF in den letzten zwei Jahren kaum verändert. Um die beiden verbleibenden Thesen würdigen zu können, sollen zunächst Sinn und Zweck des Rohstoffprogramms und die unterschiedlichen Konzeptionen seiner Realisierung gemäß den Vorstellungen der Gruppe der 77, der Industrieländer ${ }^{9}$ und der Gruppe D (COMECON-Länder) untersucht werden.

\section{Sinn und Ziele des Integrierten Rohstoffprogramms}

Die Forderungen der Entwicklungsländer nach einem Integrierten Rohstoffprogramm wovon der CF das wesentlichste Teilstück ist - müssen auf dem Hintergrund ihres nach wie vor weit überwiegend von Rohstoffen getragenen Exports gesehen werden (wobei sie generell erheblich exportabhängiger als die Industrieländer sind) und der starken Abhängigkeit der öffentlichen Haushalte - mangels anderer Einnahmen - von Exportsteuern bzw. Einkünften aus den ganz oder partiell verstaatlichten Rohstoffsektoren. Die Rohstoff preise sind nun aber nachfrage- oder angebotsbedingt (Wetter, lange Anbauzyklen) starken zyklischen

\footnotetext{
7 Vgl. Vorwärts v. 31. 3. 77, Ja zum Rohstoffprogramm.

8 Vorwärts v. 27. 10. 77, Nord-Süd-Dialog.

9 Bulletin v. 3. 11. 77, Freier Welthandel als Bedingung für Fortschritt und Entwicklung.

9a Wenn im folgenden immer wieder generalisierend von Industrieländern und Entwicklungsländern bzw. den ,,77“ die Rede ist, so soll damit keineswegs suggeriert werden, es gäbe innerhalb dieser Gruppen nicht z. T. erhebliche Differenzen.
} 
Preisschwankungen unterworfen, was in der Regel auch die Exporteinkünfte und damit die öffentlichen Einnahmen stärkeren Schwankungen unterwirft.

Der Gedanke des CF setzt an diesem Punkt an und will durch die Anlage von Rohstofflagern (engl. Bufferstocks) bei zehn lagerfähigen Rohstoffen (den sog. ,,core commodities“ des Rohstoffprogramms) (Baumwolle, Jute, Hartfasern, Kupfer, Zinn, Kaffee, Kakao, Tee, Zucker, Kautschuk) die Preisschwankungen dadurch begrenzen und innerhalb eines bestimmten Korridors halten, daß bei fallenden Preisen Rohstoffe aufgekauft und in Boomzeiten wieder verkauft werden. Durch die beim Verkauf realisierten Gewinne soll sich der CF im wesentlichen selbst tragen. Nötigenfalls werden die Bufferstock-Operationen abgestützt durch Exportquoten, die den einzelnen Produzenten auferlegtwerden. Ziel ist die Stabilisierung der Rohstoffpreise auf einem Niveau, das ,,lohnend und gerecht für die Erzeuger und fair für die Verbraucher ist" und das reale Einkommen der Entwicklungsländer erhält und verbessert ${ }^{10}$.

Dadurch soll auch vor allem eine Verstetigung des wirtschaftlichen Planungs- und Entwicklungsprozesses erreicht werden, der bislang durch das Ausbleiben von Deviseneinkünften entweder unterbrochen wurde oder die Entwicklungsländer weiter in die Verschuldung trieb ${ }^{11}$. Das Anbieten der Rohstoffe zu stabilen Preisen soll auch ihre Wettbewerbsfähigkeit gegenüber den (schon immer mehr oder weniger preisstabilen) Synthetika verbessern, ferner soll dadurch das Investitionsrisiko im Rohstoffsektor gemildert und den Entwicklungsländern Mittel in die Hand gegeben werden, um langfristige Maßnahmen der Veränderung und Verbreiterung der Produktionsstruktur (Stichwort: Diversifikation) selbst in die Hand nehmen zu können. An dieser Diversifikation setzen die ,,anderen Maßnahmen“ des Gemeinsamen Fonds an. Von den 18 Rohstoffen, die der CF umspannen soll, sind mindestens acht nicht lagerfähig (Fleisch, Bananen, Holz, Mangan, Phosphat, Bauxit, Eisenerz, Olsaaten). Für sie im besonderen, aber auch für die lagerfähigen Rohstoffe werden Maßnahmen der horizontalen (Produktion anderer Rohstoffe) und vertikalen Diversifikation (Verarbeitung der Rohstoffe im eigenen Land) der Produktivitätserhöhung, der Verbesserung des Marktzugangs, der Marktstrukturen, des Transports und der Verteilung ins Auge gefaßt ${ }^{\mathbf{1 2}}$.

\subsection{Unterschiedliche Konzeptionen eines Common Fund}

Der Gemeinsame Fonds wird nach den Vorstellungen aller Ländergruppen aus einzelnen, zwischen Rohstoffkonsumenten- und -produzentenländern ausgehandelten Rohstoffabkommen bestehen, die ihre operativen Entscheidungen (im wesentlichen Rohstoffan- und -verkäufe, Festsetzung der Lagergröße und der Interventionspreise) selbst und unabhängig vom Management des zentralen CF treffen. Mittels einmalig zur Verfügung gestellten Startkapitals sollen sich der CF und die einzelnen Rohstoffabkommen (im folgenden: ICAs = Individual Commodity Agreements) durch An- und Verkäufe von Rohstoffen finanziell selbst tragen und ein Nettoeinkommen erwirtschaften. Ubereinstimmung besteht auch darüber, daß zur Finanzierung der ,,anderen Maßnahmen“ ein zweiter Schalter eingerichtet wird, der Mittel zu besonders vergünstigten Bedingungen zusagen soll.

\footnotetext{
10 Vgl. Entschließung der IV. Welthandelskonferenz zum Rohstoffprogramm v. 30. Mai 1976, in: Entwicklungspolitik, Materialien Nr. $53 / 1976$.

11 Ebenda sowie etwa TD/B/IPC/CF/2; TD/IPC/CF/CONF/L. 2; TD/B/IPC/CF/4, Annex I.

12 Ebenda sowie die Darstellungen bei D. Kebschull etal., Dasintegrierte Rohstoff programm, Hamburg, 1977; S. Baron et al., Internationale Rohstoff politik, Tübingen, 1977; M. Tietzel, Internationale Rohstoffpolitik, Bonn-Bad Godesberg, 1977; O. Matzke, Rohstoff-Fonds - Utopie und Wirklichkeit, DIHT, Bonn, Mai 1977.
} 


\subsubsection{Ressourcenpool oder zentrale Finanzquelle}

Dissens besteht hingegen darüber, ob der CF in der von den , 77 “ vorgeschlagenen Form einer zentralen Finanzquelle oder in der Art des von den Industrieländern propagierten Ressourcenpools errichtet werden soll. Dies ist auch gleichzeitig der Hauptstreitpunkt. Bekanntlich fordert die Gruppe der 77 einen CF, der als Hauptfinanzquelle der ICAs und der anderen Maßnahmen fungieren soll, daher vor diesen und unabhängig von ihnen errichtet werden soll. Durch die so schon vorab vorhandenen Finanzmittel des CF hofft man, die Verhandlungen über den Abschluß von einzelnen Rohstoffabkommen beschleunigen zu können. Die Finanzbedürfnisse des CF (anfänglich $3 \mathrm{Mrd}$. Dollar, später $6 \mathrm{Mrd}$.) sollen durch zinslose Kapitalsubskriptionen der Mitgliedsregierungen (anfänglich $1 \mathrm{Mrd}$. Dollar, später $2 \mathrm{Mrd}$.) und doppelt so umfangreiche Anleihen bei internationalen Organisationen und vor allem auf internationalen Kapitalmärkten sowie (freilich marginal) durch Gewinne der Bufferstock-Operationen abgedeckt werden. Von diesem CF können die ICAs Kredite zur Bufferstock-Finanzierung beantragen, wobei die zu entrichtenden Zinsen möglichst niedrig liegen sollen ${ }^{\mathbf{1 3}}$.

Der Vorschlag der westlichen Industrieländer sieht hingegen einen CF vor, der als eine den ICAs nachgeordnete Institution ohne eigene Finanzmittel und ohne entscheidende Managementfunktion gedacht ist. Das Finanzvolumen des CF soll allein von der Zahl und der Größe der zustande kommenden Rohstoffabkommen abhängen, die unabhängig voneinander zwischen Produzenten- und Konsumentenländern ausgehandelt werden und die dann einen erheblichen Teil (75\%) ihres maximalen Finanzbedarfs selbst aufbringen und gegen marktnahe Zinsen beim CF deponieren würden. Dieser funktioniert als eine Art Sparkasse und stellt den ICAs zusätzlich zu ihren Einlagen noch weitere $25 \%$ ihres Finanzbedarfs automatisch und ebenfalls gegen marktnahe Zinsen zur Verfügung, wenn die ICAs diese Mittel benötigen. Sind die bisher genannten Mittel erschöpft, so soll der CF gegen Garantien noch einmal weitere $25 \%$ des Finanzbedarfs alles ICAs auf den Kapitalmärkten aufnehmen dürfen ${ }^{14}$.

Die Kritik der ,,77“ an diesem Vorschlag entzündet sich an folgenden Punkten:

a) Ein CF ohne eigenes Startkapital würde den Abschluß von ICAs nicht erleichtern.

b) Die von Gruppe B vorgeschlagene Finanzierungsstruktur würde weder die finanzielle Bürde für die Mitgliedsländer minimieren noch die Kreditbeschaffungsmöglichkeiten des CF maximieren.

c) Der Anreiz für die ICAs, an einem derartigen CF teilzunehmen sei gering, da sie dann ebenso leicht ihren Finanzbedarf an internationalen Kapitalmärkten decken könnten.

d) Wenn aber nicht alle ICAs am CF partizipieren würden, sei bei dem Vorschlag der Gruppe B auch nicht die Teilnahme aller Mitgliedsländer gewährleistet.

e) Andere Maßnahmen als den Aufbau von Rohstofflagern sehe dieser Vorschlag nicht vor. Von diesen Argumenten können wir das letzte ausschließen, da sich die Finanzierung der anderen Maßnahmen durchaus, wenn auch in anderer Form (s. u.), mit dem Vorschlag der Industrieländer vereinbaren läßt. Auf ihren harten Kern gebracht bedeuten die Einwände der , ,77“:

1. Der Vorschlag der Gruppe B würde die Entwicklungsländer erheblich teurer kommen, da hiernach Konsumenten und Produzenten nach ihrem Welthandelsanteil bei einem be-

\footnotetext{
$13 \mathrm{Vgl}$. TD/B/IPC/CF/8; TD/IPC/CF/CONF/L. 7; TD/IPC/CF/CONF/L. 5; von der Sekundärliteratur vor allem: K. Seitz, Die Verhandlungen über einen gemeinsamen Rohstoff-Fonds, in: Wirtschaftsdienst II/1978; O. Matzke, Anhaltende Kontroverse über einen gemeinsamen Rohstoff-Fonds, in: Aus Politik und Zeitgeschichte 44/1977.

14 Ebenda.
} 
stimmten Rohstoff zu den Kosten eines entsprechenden Abkommens beitragen sollen, nach den Vorstellungen der , 77 “ aber die einzelnen Länder zum CF gemäß ihrer Leistungsfähigkeit (gemessen als Anteil am Weltbruttosozialprodukt) beitragen sollen. Dazu kommt, daß dieser Vorschlag einen wesentlich höheren Eigenkapitalanteil vorsieht, nämlich $75 \%$ der Finanzbedürfnisse der ICAs gegenüber $33 \%$ Eigenkapital(= Kapitalsubskriptionen) bei einem nach den Vorstellungen der , 77 “ konzipierten CF! Die finanzielle Belastung der Entwicklungsländer fiele beim Vorschlag der Industrieländer fünf- bis sechsmal höher aus! Betroffen würden vor allem Entwicklungsländer mit einem hohen Anteil an den Weltrohstoffausfuhren.

2. Außerdem ist der Anreiz für die ICAs zur Teilnahme am CF gering, wenn sie $75 \%$ ihrer Finanzbedürfnisse selbst aufbringen müssen und für die verbleibenden $25 \%$ marktnahe Zinsen entrichten sollen. Dann können sie in der Tat auch gleich das internationale Bankensystem zur Finanzierung angehen und würden sich damit womöglich auch noch ungebetene Einmischungen des CF in ihr internes Management ersparen (s. u.). Immerhin lag der Fremdkapitalanteil des bestehenden, freilich sehr kreditwürdigen Zinnabkommens deutlich über der von den Industrieländern für die ICAs vorgesehenen Marke von $25 \%$.

3. Durch die Mischfinanzierung des $\mathrm{CF}$ aus zinslosen Kapitalsubskriptionen vergünstigten Krediten von internationalen Organisationen und kommerziellen Krediten zu normalen Konditionen, soll der CF nach dem Vorschlag der , $77^{\prime \prime}$ den ICAs sehr günstige Kredite (das UNCTAD-Sekretariat rechnet mit einem Zinssatz von 4,5 \%) gewähren können. Nach den Vorstellungen der Industrieländer sollen die ICAs aber marktnahe Zinsen (also mindestens den doppelten Satz) bezahlen. Auch dadurch wird der Anreiz für die ICAs zur Teilnahme am CF geschmälert und damit das ganze Unternehmen in Frage gestellt. Die Einwände der Industrieländer gegen den Vorschlag der Entwicklungsländer besagen, daß durch das vorgeschlagene Verhältnis von Fremd- zu Eigenkapital die finanzielle Lebensfähigkeit des CF empfindlich gefährdet würde, sofern sich überhaupt die internationalen Kapitalmärkte zur Kreditierung derart hoher Beträge bei so dünner Eigenkapitaldecke bereit finden würden. Dieses Argument ist freilich etwas vorgeschoben, da die Lebensfähigkeit des CF durch zinslose Kapitalsubskriptionen und vergünstigte Kredite internationaler Organisationen ja erheblich verbessert wird und man zudem das Urteil über die Kreditwürdigkeit getrost den Kapitalmärkten überlassen kann. Ein anderes Argument trifft freilich den Kern der Befürchtungen der Gruppe B: Die sachliche und zeitliche Trennung der Finanzierung des CF und der Verhandlungen über einzelne Rohstoffabkommen, heißt es, sei dem Abschluß ,,gesunder" Abkommen abträglich und führe zur Ressourcenverschwendung. Würden jedoch erst nach und im Maße von Rohstoffabkommen Mittel zur Verfügung gestellt, seien gesunde Basis und operative Disziplin eines CF gewährleistet ${ }^{17}$. Gemeint ist damit, daß sich ein Land wie z. B. Malaysia bei einem Anteil am Weltexport von Kautschuk in Höhe von 53,1 \% Vor- und Nachteile eines Kautschukabkommens genauer besehen wird, wenn es gemäß seinem Handelsanteil bei diesem Gut zu Zahlungen veranlagt wird, als wenn für dieses Abkommen bereits Mittel aus einem großen Topf verfügbar sind, zu dem Malaysia (nach anderem Aufbringungsschlüssel) nur zum geringsten Teil beitragen wird ${ }^{18}$. Höhere Eigenleistungen lassen auch eher ein verantwortliches Rohstoffmanagement erwarten, so die Ansicht der Gruppe B, als stark vergünstigte Mittel aus hauptsächlich fremden Quellen.

15 TD/IPC/CF/CONF/L. 7/ Add. 1, S. 4 f.

$16 \mathrm{Vgl}$. hierzu einschlägige Abhandlungen zur Außenwirtschaftspolitik wie etwa W. Glastetter, Außenwirtschaftspolitik, Köln, 1975.

17 TD/B/IPC/CF/6, Annex 1, S. 6; TD/IPC/CF/CONF/L. 7, S. 6 f.; TD/IPC/CF/CONF/L. 7/ Add. 1, S. 7.

18 Beispiel nach O. Matzke, Anm. 12, S. 50; TD/IPC/CF/8, S. 3. 
Die Position der sozialistischen Länder ist ähnlich rigide, da nach ihren Vorstellungen die ICAs ebenfalls die Hauptquelle der CF-Finanzen darstellen sollen, diese aber eher noch weniger als beim Vorschlag westlicher Industrieländer durch Regierungsbeiträge aufgebracht werden sollen. Zudem betonen die sozialistischen Staaten, daß über das Finanzvolumen welches ihnen offenbar in der geplanten Höhe zu hoch erscheint - erst nach der Klärung der einbegriffenen Rohstoffe befunden werden könne ${ }^{\mathbf{1 9}}$. Wie vom UNCTAD-Sekretariat zu erfahren, scheint sich beim Verhältnis von Eigen- zu Fremdkapital die Möglichkeit eines Kompromisses abzuzeichnen. Offenbar würden sich die Entwicklungsländer auch mit einem geringeren Anfangskapital des CF bzw. mit einem geringeren Anteil der Regierungsbeiträge im Verhältnis zu den kommerziell aufgenommenen Krediten (1:1 statt 2:1) zufriedengeben. $\mathrm{Zu}$ diesem Verhältnis und zu wenigstens symbolischen Eigenmitteln (100 bis 200 Mill. Dollar) würde sich mittlerweile auch die Bundesregierung - zumindest das Auswärtige Amt - verstehen, nachdem auch schon die Vereinigten Staaten weitreichendere Vorschläge lanciert hatten ${ }^{20}$. Dies zeigt an, daß auch die Industrieländer den anfänglich vorgeschlagenen Eigenkapitalanteil für zu hoch halten, und eröffnet gleichzeitig eine Chance der Konfliktbeilegung. Ubrigens bestehen auch Bedenken hinsichtlich möglicher Verantwortungslosigkeit bei zu geringen Eigenleistungen m. E. allenfalls teilweise zu recht, denn bei den Rohstoffabkommen würden Konsumenten und Produzenten gleiches Stimmrecht haben, so daß also unverantwortliche Rohstoffoperationen verhindert werden könnten. Auch bei den von den , 77 “ anders gewünschten Stimmverhältnissen im CF wären gewisse Sicherungen (s. u.) gegen ,,Verantwortungslosigkeit“ eingebaut.

Eine andere Gefahr ist allerdings, daß sich bei einem nach den Vorstellungen der , 77 “ konzipierten Fonds die Rohstoffabkommen, die zuerst abgeschlossen werden, bevorzugt aus der schon vorhandenen Finanzmasse des CF bedienen bzw. beim Zustandekommen von weniger als 18 Rohstoffabkommen (wovon 10 mit Ausgleichslagern) die verbleibenden ICAs sich die Gesamtmittel aneignen. Diese Bedenken werden vor allem vergrößert, daß sich die , ,77“ bisher weigerten, Höchstgrenzen der Kredite des CF an ein einzelnen Rohstoffabkommen zu akzeptieren (vorgeschlagen werden statt dessen interne Regelungen), um Spekulationen abzuschrecken, die etwa bei Erreichen des Kreditlimits eines ICA durch Rohstoff verkäufe weiteren Preisverfall herbeiführen könnten. Damit wächst aber die Gefahr, daß sich eine Minderheit von ICAs alle Mittel des CF aneignet. Hier wäre Raum für eine mögliche und sinnvolle Forderung der Industrieländer, sei es nach Höchstlimits, sei es nach einer Kapitalausstattung des CF im Maße von abgeschlossenen Rohstoffvereinbarungen.

\subsubsection{Die Finanzierung anderer Maßnahmen}

Wie schon erwähnt, soll der CF nach den Vorstellungen der Gruppe „77” neben seiner hauptsächlichen Aufgabe, der Finanzierung von Ausgleichslagern, auch andere rohstoffpolitische Maßnahmen finanzieren, und zwar:

a) bei Rohstoffen, die sich ständig fallenden Preisen und steigender Substitution durch Synthetika gegenübersehen; hier ist an eine Verbesserung der Konkurrenzfähigkeit durch produktivitätssteigernde Maßnahmen gedacht;

\footnotetext{
19 Vgl. TD/IPC/CF/CONF/L. 7, S. 9.

20 Interview bei der UNCTAD; siehe auch O. Matzke, Der Gemeinsame Fonds in einer Sackgasse, NZZ v. 27.5 .78$.

21 Sehr kritisch hierzu: O. Matzke, Anm. 13; zum Vorschlag der Industrieländer vgl. TD/IPC/CF/CONF/L. 5 sowie: NZZ v. 26.10. 77, Die Position der westlichen Industrieländer zu einem gemeinsamen Rohstoff-Fonds.
} 
b) bei Rohstoffen, wo ein ständiger Angebotsüberhang zur Akkumulation von Lagervorräten führt; hier sollen Diversifikationsprojekte finanziert werden.

Profitieren sollen von 1 bis 1,5 Mrd. Dollar, die für derartige Maßnahmen vorgesehen sind, vor allem die nicht-lagerfähigen Rohstoffe, da die anderen ja schon auf dem Wege von Bufferstocks unterstützt werden ${ }^{22}$. Die westlichen Industrieländer sind der Ansicht, daß diese Maßnahme besser über die bestehenden internationalen Organisationen (vor allem die Weltbank und die regionalen Entwicklungsbanken) und über die bilaterale Entwicklungshilfe zu finanzieren wären, wobei sie sich allerdings allmählich gesprächsbereiter zeigen. Sie sehen die anderen Maßnahmen als Domäne der Entwicklungshilfe an, da derlei Investitionen sehr langfristiger Natur sind und auf eine schnelle Amortisation der eingesetzten Mittel nicht gehofft werden darf. Bei einer Finanzierung dieser Maßnahmen durch den CF fürchten sie daher auch für dessen kommerzielle Lebensfähigkeit ${ }^{23}$. Ein nicht genanntes Motiv der Gruppe $B$ ist die Tatsache, daß sie bei der bilateralen Entwicklungshilfe und bei der Weltbank die Mittelvergabe natürlich wesentlich stärker kontrollieren könnten als bei einem zweiten Schalter des CF.

Die sozialistischen Staaten sorgen sich vor allem über den Umfang der ins Auge gefaßten anderen Maßnahmen und wollen sie nur innerhalb von ICAs finanziert wissen ${ }^{\mathbf{2 4}}$.

Gegen eine Finanzierung der anderen Maßnahmen durch die existierenden internationalen Organisationen wenden die , 77 “ ein, daß

- deren Länder - statt Rohstofforientierung nicht die Bearbeitung jener Probleme erlaube, die sich das Integrierte Programm vornehme;

- die Mitgliedschaft in den bestehenden I. O. nicht universal sei;

- diese nur an Regierungen, nicht aber an ICAs Kredite geben könnten;

- diese nicht die übergreifende Koordination aller rohstoffbezogenen Maßnahmen auf dem Hintergrund eines umfassenden Uberblicks über den gesamten Weltrohstoffhandel leisten könnten;

- die Delegation an die bestehenden I. O. den CF seiner ,,katalytischen“ Funktion berauben würde, d. h., der Aufkauf von Lagervorräten bei Rohstoffen, für die noch keine Abkommen existieren, wäre ausgeschlossen. Zudem gäbe es dann Schwierigkeiten bei der Identifikation abkommensbegleitender und -unterstützender Vorhaben ${ }^{\mathbf{2 5}}$.

Diese Argumente sind nur teilweise stichhaltig. Zunächst einmal ist jede größere bi- oder multilaterale Entwicklungshilfeorganisation sowohl nach nationalen wie nach sektoralen Gesichtspunkten gegliedert. Es ist völlig unerfindlich, warum die schon bestehenden Rohstoffabteilungen der Entwicklungsbanken, des UNDP und der bilateralen Organe nicht erweitert und mit qualifizierten Fachkräften ausgestattet werden könnten, zumal die Weltbank und die regionalen Entwicklungsbanken bereits 3 Mrd. Dollar (für 1975 bis 1979) für solche anderen Maßnahmen eingeplant haben. Auch geben die Entwicklungsbanken durchaus Kredite an Nicht-Regierungen, nämlich an Entwicklungsfinanzierungsgesellschaften. Der mangelnden Universalität der I. O. könnte abgeholfen werden, indem diese nur die Projektausführung übernehmen, beim CF aber Projektzusage und -finanzierung verbleiben. Genau dies sind ja auch die Vorstellungen des UNCTAD-Sekretariats und der ,77“. Ungenannt wie oben bleibt die Tatsache, daß die Entwicklungsländer in den bestehenden I. $\mathrm{O}$. (im Gegensatz zum CF) majorisiert werden können.

Berechtigt sind die Argumente der , 77 “ aber insoweit, als tatsächlich einige u. E. für die Sinnhaftigkeit des gesamten Rohstoffprogramms entscheidende Aufgaben von den beste-

$22 \mathrm{Vgl}$. TD $/ \mathrm{B} / \mathrm{IPC} / \mathrm{CF} / \mathrm{L}$. 3.

23 Vgl. TD/B/IPC/CF/6, S. 8 ff.; TD/B/IPC/CF/8, S. 9; TD/IPC/CF/CONF/L. 7/Add. 1, S. 6.

$24 \mathrm{TD} / \mathrm{B} / \mathrm{IPC} / \mathrm{CF} / 6$, S. 9 ; TD/B/IPC/CF/8, S. 4; TD/IPC/CF/CONF/L. 7/Add. 1, S. 9.

$25 \mathrm{TD} / \mathrm{IPC} / \mathrm{CONF} / \mathrm{L}$. 7/Add. 1, S. $2 \mathrm{f}$. 
henden internationalen Organisationen nicht in ausreichendem Maße wahrgenommen werden können. Wie bereits ausgeführt, sind von den 18 Rohstoffen des CF acht nicht lagerfähig, vermutlich werden im Laufe der Abkommensverhandlungen sich auch noch weitere Rohstoffe als weniger geeignet für Bufferstock-Abkommen erweisen. Für diese Rohstoffe werden die anderen Maßnahmen die einzig praktikable Unterstützungsform darstellen. Dazu kommt, daß der Rohstoffsektor weltweit betrachtet ,,zu den am wenigsten dynamischen Sektoren " gehört ${ }^{\mathbf{2 6}}$. So gehört es denn auch zum Credo einer ganzen Generation von Wirtschaftswissenschaftlern ${ }^{27}$, den Entwicklungsländern die Weiterverarbeitung ihrer Rohstoffe im Lande bzw. die Produktion arbeitsintensiv gefertigter Halb- und Fertigprodukte statt der zunehmend unattraktiv werdenden Rohstofförderung zu empfehlen. Was läge nun allerdings näher, als diesen Umstrukturierungsprozeß durch großzügig unterstützte Diversifizierungsfonds zu erleichtern, d. h. den lokalen Produzenten entsprechende Start- und Úberbrückungshilfen zu gewähren? Diese Hilfen wären am besten global und mit den Bufferstock-Operationen abzustimmen, um erneute Fehlinvestitionen zu vermeiden, weshalb eine Zentralisation dieser Aufgaben beim CF durchaus Sinn macht. Sodann wird auch das Funktionieren der Bufferstock-Operationen verbessert, wenn neben der Preisstabilisierung auch die Konkurrenzfähigkeit der Produkte (durch Produktivitätsverbesserung) erhöht werden kann oder wenn zusätzlich notwendig werdende Exportbeschränkungen dadurch für die Produzenten erträglicher werden, daß sie Mittel für die Diversifikation erhalten. Ein Mangel an Entgegenkommen bei den anderen Maßnahmen wird gerade die in der Bundesrepublik beschworene Gefahr der Uberproduktion bei manchen Rohstoffen sicher nicht mindern $^{28}$. Freilich können diese Maßnahmen nicht wie die Bufferstock-Operationen hauptsächlich mittels kommerzieller Kredite finanziert werden, da sie in der Tat langfristiger Natur sind. Es muß überdies auch fraglich erscheinen, ob die von der UNCTAD eingeplanten Mittel für diese Maßnahmen tatsächlich ausreichen, um über Jahre hinaus umfangreiche Diversifikationsprojekte und Überbrückungshilfen zu finanzieren. Mittlerweile zeichnet sich nach Aussagen des UNCTAD-Sekretariats in der Frage der anderen Maßnahmen ein Kompromiß ab, der ihre Finanzierung über einen Zweiten Schalter des CF auf der Basis freiwilliger und zinsloser Regierungszusagen vorsieht ${ }^{29}$. Diese ,kleine“ Lösung wäre m. E. aber auch für die Industrieländer ein schlechtes Geschäft, da bei dem dann zu erwartenden geringen Volumen des ,Zweiten Schalters“ echte und langfristige Lösungen des Rohstoffproblems retardiert würden. Zum anderen würde diese Lösung auch die Konsensfähigkeit des von Gruppe B vorgeschlagenen ,,Ressourcenpools“ noch weiter schmälern. Wie bekannt, würden von den anderen Maßnahmen vor allem die Länder ohne attraktive und lagerfähige Rohstoffe profitieren. Da diese Länder - es handelt sich hauptsächlich um afrikanische Staaten - hinsichtlich anderer Aspekte des CF eher zu den Gemäßigten unter den ,,77“ zählen, läge es im aufgeklärten Interesse der Industrieländer, diese Staaten durch großzügige Konzessionen bei den anderen Maßnahmen für ihr Pooling-Konzept zu gewinnen ${ }^{\mathbf{3 0}}$. Denn die Vorschläge der Gruppe B wurden nicht zuletzt deswegen immer verworfen, weil sie keinen Raum für die anderen Maßnahmen lassen ${ }^{31}$.

26 Vgl. O. Matzke, UNCTAD IV und danach, in: A us Politik und Zeitgeschichte 37/1976, S. 19

27 Siehe dazu nur die Jahrgänge der Zeitschriften ,Die Weltwirtschaft" und ,,Weltwirtschaftliches Archiv“ sowie den Sammelband: H: Giersch (Hrsg.), The International Division of Labour, Tübingen, 1974.

28 Ähnlich auch die Stellungnahme der Kirchen auf dem Hearing des BT-Ausschusses für wirtschaftliche Zusammenarbeit am 23. und 25. Mai 1977, in Th. Dams/G. Grohs (Hrsg.), Kontroversen in der internationalen Rohstoff politik, München 1977 sowie K. Seitz, Anm. 13.

29 Interview bei der UNCTAD.

30 So auch K. Seitz, a. a. O.; Robert R. Cox, Der Gemeinsame Fonds - eine Zwischenbilanz, in: Vereinte Nationen, 4/1977 und The Economist v. 12. 11. 77, Stabilizing Commodity Prices: Can Rich and Poor Agree?

31 TD/IPC/CF/CONF/L. 7/dd. 1, S. 4; TD/IPC/CF/CONF/L. 7, S. 5. 


\subsubsection{Interventionen außerhalb von Rohstoffabkommen}

Klarer Dissens besteht zwischen westlichen und östlichen Industrieländern und den , $77^{\prime \prime}$ in der Frage von Marktinterventionen des CF bei Rohstoffen, für die keine bzw. noch keine Abkommen bestehen. Der CF soll nach den Vorstellungen der Entwicklungsländer in schwierigen Situationen (bei Preiseinbrüchen) diese Rohstoffe auf Lager nehmen können und sie bis zum Abschluß eines entsprechenden Abkommens zwecks Preisstabilisierung verwalten.

Für diese in der Tat etwas problematisch erscheinenden Operationen - bei Nichtzustandekommen eines Abkommens müßte der CF die Lager möglicherweise unter hohen Verlusten (wenn die Preise weiter fallen) auflösen - sieht aber das UNCTAD-Sekretariat etliche Kautelen vor: einmal sollen diese Interventionen nur auf Antrag von Produzentenländern erfolgen, die zusammen mindestens 50 \% des Exports des in Frage stehenden Rohstoffes bestreiten und sofern diese zu Verhandlungen über ein Rohstoffabkommen bereit sind. Sodann soll die Intervention des CF nur auf begrenzte Zeit erfolgen bei Billigung durch eine qualifizierte Mehrheit im board of directors des CF, womit die Gruppe B die Möglichkeit zum Veto hät$\mathrm{te}^{32}$.

Die westlichen Industrieländer wenden sich gegen Vorabausgaben von ,,etlichen hundert Mill. Dollar"“ in der vagen Hoffnung, daß im Laufe der Interventionen ein Abkommen zustande kommt. Dies würde den CF in Verluste treiben und sei auch deshalb abzulehnen, weil die Tatsache, daß kein oder noch kein Abkommen zustande gekommen sei, grundsätzliche Interessendivergenzen zwischen Produzenten und Konsumenten anzeige, die nicht durch Mittelvergabe an die Produzenten gelöst werden könnten ${ }^{\mathbf{3 4}}$. In dieser Frage sind sich alle Länder außerhalb der Gruppe der ,,77“ einig - ein wirklich seltener Fall -, so daß die Realisierung dieser Vorstellungen wohl kaum zu befürchten ist.

\subsubsection{Die Autonomie der Rohstoffabkommen}

Wie schon ausgeführt, besteht hinsichtlich der operativen Autonomie der ICAs gegenüber dem CF Konsens zwischen allen Gruppen, der allerdings mehr verbaler Art ist. Fordern die westlichen Industrieländer völlige Autonomie der ICAs bezüglich der Entscheidungen über Größe und Finanzierung der Ausgleichslager, so schließen die Vorstellungen der UNCTAD - und der damit völlig identischen der , 77 “ - weitreichende Eingriffe des CF in die Politik der ICAs ein. Dies vor allem dann, wenn die intern für die ICAs vorgesehenen Kreditbeträge (s. o.) überschritten werden. Dann soll sich der CF von der Angemessenheit der Lagervorräte, der Exportquoten und anderer angebotsbeschränkender Maßnahmen, der Sinnhaftigkeit der Interventionspreise, der Effektivität des Managements und ganz allgemein der wirtschaftlichen Gesundheit der ICAs überzeugen. Diese Prüfung soll mit dem Ziel erfolgen, die unerwünschte Akkumulation von Lagervorräten zu vermeiden ${ }^{35}$. Höchstlimits der Kredite an die ICAs, die einen Teil dieser Eingriffe unnötig machen würden, werden wie gesagt von den ,„77“ abgelehnt. Erwartungsgemäß plädieren die Industrieländer hierfür aber ebenso wie für einen automatischen Zugang der ICAs zu den Krediten des $\mathrm{CF}^{36}$. In einem späteren

\footnotetext{
32 TD/B/C. 1/184, para. 50 u. 54; TD/B/C. 1/196, para. 38 u. 39; TD/B/IPC/CF/L. 5, S. 4.

$33 \mathrm{TD} / \mathrm{IPC} / \mathrm{CF} / \mathrm{CONF} / \mathrm{L} .2$, S. 4.

34 TD/B/IPC/CF/6, S. 9; TD/B/IPC/CF/8, S. $4 \mathrm{f}$

35 TD/B/IPC/CF/2, S. 9 f.; TD/B/IPC/CF/L. 5, S. 2 ff.; TD/IPC/CF/CONF/3, S. 7 f.

36 TD/IPC/CF/CONF/L. 3, S. 1.
} 
Entwurf der Gruppe der , 77 “ werden die weitgehenden Eingriffsrechte des CF partiell wieder zurückgenommen. Ob dieses Entgegenkommen sinnvoll ist, steht dahin. Die Nichteinmischung des CF in die Politik der ICAs macht nämlich nur dann Sinn, wenn sich der CF nach dem Vorschlag der Industrieländer konsituiert, d. h. die ICAs ihre Finanzmittel weitgehend selbst aufbringen. Anders, wenn der $\mathrm{CF}$ als zentrale Finanzquelle konkurrierender ICAs konzipiert ist: Hier sind Eingriffsrechte des CF, solange keine Höchstlimits für die Kredite an einzelne Rohstoffabkommen gesetzt werden, logisch und vielleicht sogar wünschenswert. Man sollte achtgeben, daß nicht, wie bei vielen Reformversuchen, zum Schluß ein CF herauskommt, der die weniger sinnvollen und kompatiblen Detailregelungen der verschiedenen Parteien in sich vereinigt.

\subsubsection{Stimmrechte im $\mathrm{CF}$}

In Anbetracht der Natur und der Ziele des CF fordern die Entwicklungsländer eine Stimmverteilung, die ihnen ,,eine entscheidende Rolle bei den Beschlüssen und beim Management des Fonds" gibt. Diese reflektiert die tiefer liegenden Bestrebungen der Entwicklungsländer nach einer Revision der Macht- und Einflußverteilung im Gefüge der alten und neuen internationalen Institutionen. Die Stimmrechte sollen folgerichtig nicht nur nach der Höhe der Kapitalsubskriptionen, sondern zu einem guten Teil nach dem Gleichheitsgrundsatz verteilt $\operatorname{sein}^{37}$. Die Industrieländer hingegen befürworten Stimmengleichheit von Produzenten und Konsumenten bei enger Anlehnung der Stimmrechte an die Kapitalbeteiligung ${ }^{\mathbf{3 8}}$.

Diese Debatte ist trotz der Aufregung, die sie verursacht hat, eher akademischer Natur, da zur Entscheidung über wichtige Fragen in den Leitungsgremien des CF qualifizierte Mehrheiten (nach Vorschlag des UNCTAD-Sekretariats 2/3- oder 3/4-Mehrheiten) erforderlich sein werden und unter ,, wichtigen Fragen" alles fallen wird, was einigermaßen von Belang ist $^{39}$. Eine qualifizierte Mehrheit würde aber den Industrieländern die Möglichkeit des Vetos einräumen.

Das Sekretariat gab in Gesprächen auch zu verstehen, daß unerachtet der Stimmrechte beispielsweise bei der Weltbank dort immer ein Konsens gefunden werde und daß sich der CF aus Gründen der Seriosität (s. u.) Auseinandersetzungen in den Leitungsgremien kaum leisten könne. Man hält zudem als Ergebnis der Verhandlungen eine Stimmverteilung von 50:50 für am wahrscheinlichsten ${ }^{40}$.

\section{Auf dem Wege zu einer Weltplanwirtschaft?}

Es fällt einigermaßen schwer, die bislang aufgeführten Differenzen zwischen den , 77 “ und den westlichen Industrieländern als den Kampf um markt- bzw. planwirtschaftliche Prinzipien zu interpretieren, wie es vorzugsweise in der Bundesrepublik geschah ${ }^{\mathbf{4}}$. Auf dem Spiele stehen im wesentlichen nur eine mehr oder weniger sorgfältige Verwendung der Mittel und mehr oder minder große Kostenbeteiligungen bzw. Einflußmöglichkeiten der einzelnen Ländergruppen. Es muß einigermaßen künstlich wirken, wenn die Grenze zur Marktwirt-

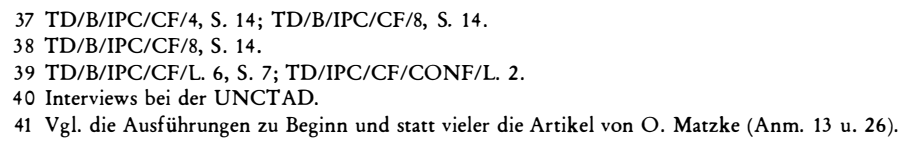


schaft dort gezogen wird, wo die ICAs Preise oberhalb des Marktgleichgewichts zu setzen versuchen, zumindest wenn diese Preise nicht dauerhaft durchgesetzt werden können ${ }^{42}$, oder dort, wo der CF neben den Einlagen der ICAs noch zusätzlich über eigene Mittel verfügt.

Das UNCTAD-Sekretariat betrachtete es immer schon als ein grobes Mißverständnis, das Ziel des CF als „,Ausschaltung der Marktkräfte“ (O. Matzke) ${ }^{44}$ zu begreifen: ,.. . . das Hauptziel des Programms ist nicht der Bruch mit der Marktwirtschaft, sondern die Verbesserung ihres Funktionierens mit höherer Stabilität, die dadurch erreicht wird, daß exzessivem Preisverfall Grenzen gesetzt werden, die bislang in diesem System stattfinden ${ }^{45}$." Dies könnte freilich noch als interessiertes Abwiegeln der UNCTAD betrachtet werden. Worauf stützen sich aber jene, die den CF als ,,superdirigistisches“ Machwerk brandmarken? Diese Kritiker vermuten:

a) daß künftig das Rohstoff preisniveau ausgehandelt bzw. administriert statt durch Angebot und Nachfrage bestimmt wird;

b) daß mit dem CF eine Fixierung der Rohstoffpreise über dem langfristig sich auf dem Markt einstellenden Gleichgewichtspreis versucht werde, um die Einkünfte der Produzenten zu verbessern;

c) daß die daraus folgende Úberproduktion zu Rohstoffhalden führe, die nach dem Muster des EG-Agrarmarktes verwaltet würden;

d) daß diese und ähnliche Probleme bald riesige Bürokratien zur Verwaltung des Rohstoffmarktes nötig machten;

e) daß die anfänglichen Eingriffe in den Markt bald durch weitergehende (Exportquoten, Abnahmeverpflichtungen) ersetzt würden ${ }^{\mathbf{4 6}}$.

Der kritische Angelpunkt bei der Debatte ist die Fixierung der Interventionspreise, auf deren Niveau die Rohstoffpreise durch die Bufferstock- Operationen der ICAs stabilisiert werden sollen. Vor allem deutsche Autoren ergehen sich gerne in Betrachtungen über die Unmöglichkeit, den künftigen Gleichgewichtspreis bei Rohstoffen vorauszuschätzen. Diese Prognosefähigkeit bestünde vor allem aber bei manipulierten Preisen nicht mehr: ,,Wenn erst einmal ein System administrierter Preise besteht, fehlt bald die Prognosebasis, da es unwahrscheinlich ist, daß administrierte Preise Gleichgewichtssituationen widerspiegeln ${ }^{47}$. "Wollten die ICA-Manager die Preise tatsächlich vollständig administrieren, müßten sie die ganze Produktion aufkaufen. Die obige Argumentation ist schon deshalb unsinnig, weil auch bislang bereits ein großer Teilder Rohstoffverkäufe (vor allem bei Bananen, Tee, Kupfer, Zinn, Mangan, Eisenerz) konzernintern bzw. mittels langfristiger Abnahmeverpflichtungen zu vorabfixierten Preisen getätigt wird, was den Markt durchaus nicht hindert, für die Restmenge einen nicht administrierten Preis zu bilden. Die Mittel des CF würden vermutlich gerade ausreichen, Ausgleichslager in Höhe von 1/6 des Jahresexports der lagerfähigen Rohstoffe zu bilden, ließen also der freien Preisbildung genügend Raum.

Berechtigt ist die obige Argumentation aber insoweit, als in der Tat der künftige Marktpreis für einen Roḩstoff nicht exakt vorauszusehen ist. Setzen die ICAs aber ihre Interventions-

\footnotetext{
42 Vgl. Anm. 9.

43 Vgl. NZZv. 26. 10.77, Die Position der westlichen Industrieländer zu einem gemeinsamen Rohstoff-Fonds(O. Matzke); O. Matzke, Anm. 13, S. 52.

44 O. Matzke, Anm. 13, S. 44; ders., Anm. 26, S. 13.

$45 \mathrm{Vgl}$. TD/B/IPC/CF/6, Annex 1, S. 7.

46 Vgl. Der Spiegel v. 17. 5. 76, Kritik von Cosmos; General Anzeiger v. 1. 6. 76, Bonn lehnt dirigistische Maßnahmen ab; FAZ v. 3. 6. 76, Friderichs und Bahr mit Nairobi zufrieden; Rheinischer Merkur v. 10. 12.76, Schöpferische Pause;FAZv. 27. 12. 76, wachsender ausländischer Druck auf die deutsche Entwicklungspolitik.

47 B. Stecher, Die internationale Rohstoffpolitik: Bisherige Erfahrungen und neue programmatische Ansätze, in: Th. Dams/G. Groh, Anm. 28, S. 45; ähnlich: M. Tietzel, Anm. 12, S. 83.
} 
preise (gegenüber dem künftigen Marktpreis) zu hoch oder zu niedrig an, so werden die Rohstoffabkommen bald funktionsunfähig, sei es durch leere Rohstofflager oder durch Erschöpfung der finanziellen Mittel, die ihnen zur Verfügung stehen. Begegnet werden kann diesem Problem nur durch periodische Neufestsetzungen der Interventionspreise, also sobald sich zeigt, daß die geltenden Preise zur Akkumulation von Vorräten bzw. zu deren Erschöpfung führen. D. Kebschull schlägt in diesem Zusammenhang stufenflexible Abkommen vor, K. Seitz plädiert für eine Preisfindung durch trial and error ${ }^{\mathbf{4 8}}$. Im UNCTAD-Sekretariat denkt man daran, den Preis an den jeweiligen Umfang der Lagermengen zu binden und, bei deren Vergrößerung etwa, entsprechende prozentuale Preisabschläge vorzunehmen $^{49}$. Das setzt aber voraus, daß sich Produzenten- und Konsumentenländer der ICAs über einen Mechanismus zur Preisneubestimmung einigen (statt nur über die Preise selbst), was angesichts historischer Erfahrungen mit Skepsis betrachtet werden kann ${ }^{50}$. Diese Skepsis hinsichtlich der technischen Durchführbarkeit ist aber etwas ganz anderes als die Behauptung, der CF suspendiere die Marktwirtschaft. Uberhaupt ist immer wieder zu beobachten, daß die Kritiker des CF sowohl den anti-marktwirtschaftlichen Charakter des Fonds hervorheben wie auch seine Funktionsunfähigkeit unterstellen. Beides kann freilich nicht logisch zur gleichen Zeit behauptet werden.

Es bleibt das Argument, die ICAs könnten ihre Interventionspreise zu hoch ansetzen, um den Entwicklungsländern Mehreinnahmen zu verschaffen und die dann daraus folgenden Úberschüsse müßten nach dem Muster des EG-Agrarmarkts verwaltet werden. Begünstigt wird diese Vermutung dadurch, daß die Forderungen nach einem Rohstoff programm historisch im Kontext mit Plänen entstanden sind, für Rohstoffe Kartelle nach dem Muster der OPEC zu bilden bzw. die Rohstoff preise an einen Index der von Entwicklungsländern importierten Fertiggüter (= Indexierung) zu binden. Von diesen Plänen ist freilich seit UNCTAD IV nicht mehr die Rede (was vor allem deutsche wirtschaftswissenschaftliche Institute nicht daran gehindert hat, sich in Gutachten für die Bundesregierung auch später noch weitschweifig über deren Realisierungschance zu verbreiten). Dazu kommt, daß Verlautbarungen der UNCTAD und der , „77“'von einer Stabilisierung auf einem Niveau sprechen, daß , ,lohnend und gerecht für die Erzeuger und fair für die Verbraucher" ist und daher Preiserhöhungsabsichten vermuten lassen. Mit dieser Formulierung soll aber nicht das moralische Problem des gerechten Preises gelöst werden (so etwa O. Matzke) ${ }^{51}$, es ist vielmehr die seit Jahrzehnten übliche Floskel internationaler Rohstoffabkommen ${ }^{\mathbf{5 2}}$.

Preiserhöhungen über den Marktrend sind schon deshalb schwierig, weil die Konsumenten bei den ICAs gleichberechtigt über die Preise mitentscheiden. Die Befürchtungen sind auch deshalb nicht stichhaltig, weil der CF mit einer begrenzten Summe zur Marktintervention ausgestattet wird und sich finanziell selbst tragen muß. Es wäre daher vom Management des $\mathrm{CF}$ ausgesprochen töricht, die nur einmal zu Beginn erfolgende Kapitalausstattung durch Halten unrealistischer Preise und Auftürmen endloser Lagervorräte zu verschleudern. Ziel des CF ist ja nicht eine Einkommensgarantie für die Produzenten (wie beim EG-Agrarmarkt), wozu jährlich mittels erheblicher Summen aus öffentlichen Haushalten beliebige Ausstoßmengen aufgekauft werden, sondern die Preisstabilisierung entlang des langfristigen Markttrends. Dies wird auch mehr und mehr von deutschen Autoren anerkannt. ${ }^{53}$

48 Interview bei der UNCTAD.

49 D. Kebschull, Stufenflexible Abkommen als gangbarer Weg, in: Wirtschaftsdienst V/1976; K. Seitz, a. a. O.

50 Vgl. M. Tietzel, Anm. 12, S. 82 f.; O. Matzke, Anm. 13, S. 52

51 Vgl. O. Matzke, Anm. 12, S. 8 f.

52 Interview bei der UNCTAD.

53 Vgl. K. Seitz, a. a. O.; D. Kebschull, Lagerhaltung, Harmonisierung der Lagerhaltungspolitik und Preispolitik, in Th. Dams/G. Groh, Anm. 28, S. 97 ff. 
Der CF könnte die Rohstoffpreise bei lang anhaltenden Nachfragerückgängen auch nicht durch Anhäufungen von Lagervorräten halten, sondern allenfalls durch zusätzlich den Produzenten auferlegte Exportquoten, die das Rohstoffangebot nachhaltig mindern. Dies wird auch vom UNCTAD-Sekretariat offen angesprochen. ${ }^{54}$ Exportquoten stellen zwar in der Tat direkte Eingriffe in den Wirtschaftsablauf dar, zu bedenken ist dabei aber zweierlei: a. Normalerweise wird es durchaus als Wesensmerkmal der Marktwirtschaft betrachtet, daß die Produzenten, wenn sie schon nicht ihren Verkaufspreis fixieren können (wie bei Rohstoffen der Fall), wenigstens die von ihnen zu einem bestimmten Preis angebotener. Mengen bestimmen. Dazu sind aber die rohstoffexportierden Länder bislang weder nach außen (wegen des aus ständigem Devisenmangel folgenden Exportzwanges) noch nach innen (wegen der oft nicht vorhandenen nationalen Rohstofflager und der schwachen Durchsetzungsfähigkeit der Administration gegenüber den Produzenten) in der Lage. Die Finanzierung nationaler Lager, vor allem aber die Rohstoffankäufe durch die ICAs und die nunmehr von allen Konsumenten- und Produzentenländern überwachten Exportquoten (s. u.) könnten diese Aufgabe wesentlich erleichtern.

b. Je großzügiger die Ausgleichslager ausgestattet sind je schneller Diversifikationprojekte bei Rohstoffen, die sich anhaltenden Nachfragerückgängen gegenübersehen, in Angriff genommen werden, desto mehr kann auf ,,marktfremde“ Eingriffe wie Exportquoten verzichtet werden. ${ }^{56}$ Das Problem der Exportquoten liegt vor allem bei der Fixierung der Anteile für die einzelnen Produzentenländer, da diese Anteile sich an der Produktion der vergangenen Jahre orientieren. Sie müßten bei einer Änderung der Konkurrenzposition und bei neuen Nachfragebedingungen (wenn etwa die Konsumenten plötzlich andere Kaffeesorten bevorzugen) entsprechend angepaßt werden. Zudem sollten die Quoten zugunsten der ärmeren Länder etwas höher ausfallen, da diese schlechter auf Deviseneinnahmen verzichten können. Diese und andere Erfordernisse bedingten auch schon bei den bisherigen Abkommen ein hartes Ringen um die Exportquoten und schufen Anreize (besonders bei Preisauftrieb des in Frage stehenden Rohstoffes) sich unter Verletzung der Solidarität Extragewinne anzueignen. ${ }^{57}$ Dazu kommt, daß sich auch die rohstoff produzierenden Industrieländer und die sozialistischen Staaten den Exportquoten unterwerfen müßten und diese in den Entwicklungsländern auch intern durchzusetzen sind. ${ }^{58}$

Diese Schwierigkeiten und die vorgebrachten marktwirtschaftlichen Bedenken legen eigentlich Konzessionen in Richtung auf großzügige Kapitalausstattung der Ausgleichslager und der Diversifizierungsmaßnahmen nahe. Auch andere Uberlegungen vermögen Befürchtungen hinsichtlich der marktzerstörenden Qualität eines CF zu zerstreuen. Bei allen Beteiligten herrscht nämlich Ưbereinstimmung darüber, daß der CF und die ICAs als finanziell und kommerziell lebensfähige Organe funktionieren sollen. Strittig sind dabei nur die Zinssätze (der CF-Kredite und der ICA-Einlagen), die diese Lebensfähigkeit garantieren und Nettoprofite des CF ermöglichen können. ${ }^{59}$ Das UNCTAD-Sekretariat betont immer wieder die Notwendigkeit gesunder finanzieller Prinzipien für den zu schaffenden Gemeinsamen Fonds: ,,Auf den internationalen Kapitalmärkten würde der CF mit vielen anderen privaten und öffentlichen Gläubigern konkurrieren müssen und wird daher seine relativen Verdienste

\footnotetext{
54 Vgl. etwa TD/B/IPC/CF/4, Annex 1.

55 Vgl. G.K. Helleiner, A Bad Case: Grubel on the New International Economic Order, in: Weltwirtschaftliches Archiv, Bd. CXIV, 1978 , S. 166.

$56 \mathrm{Vgl}$. TD/B/IPC/CF/4, S. 8.

57 D. Kebschull, Anm. 53, S. 85; O. Matzke, Anm. 12, S. 11 u. 23; H. Glismann et al., Ókonomische Implikationen der ,Neuen Weltwirtschaftsordnung", Kieler Diskussionsbeiträge Nr. 46, Kiel, 1976, S. 22.

58 Interview bei der UNCTAD sowie O. Matzke, Anm. 12, S. 58.

59 Zur Debatte hierüber TD/B/IPC/CF/8, S. $10 \mathrm{ff}$.
} 
auf der Basis finanzieller Gesundheit und anderer Garantien zu demonstrieren haben, um dieselbe hohe Kreditwürdigkeit wie andere internationale Institutionen zu erlangen. "60 Dies sei vor allem während der Anfangsjahre wichtig. Damit wird aber deutlich: Ein Fonds, der auf internationalen Kapitalmärkten die gleiche Bewertung wie die Weltbank erreichen will (AAA-rating $=$ höchste Stufe der Kreditwürdigkeit) bei relativ hohem Anteil des Fremdkapitals, wird sich zwangsläufig eines seriösen Geschäftsgebarens befleißigen müssen. Der CF, so auch die Meinung im UNCTAD-Sekretariat, wird von daher eher eine konservative Organisation werden. Von daher sind zusätzlich Befürchtungen wegen der von der Gruppe der 77 gewünschten Stimmverteilung nur wenig am Platze, wäre doch der CF bei Anleihen auf westlichen Kapitalmärkten ( wo auch sonst) auf die mitarbeit der Industrieländer angewiesen. ${ }^{61}$

Angestoßen durch Úberlegungen in Kreisen amerikanischer Wirtschaftswissenschaftler (N. Kaldor, J. R. Behrman) und der amerikanischen Administration, registrierte man auch langsam in der Bundesrepublik, daß Rohstoffabkommen durchaus auch Vorteile für die Industrieländer haben könnten. Die Fluktuationen der Rohstoffpreise wirken nämlich durch ihr direktes Durchschlagen auf die Deviseneinkommen der Entwicklungsländer prozyklisch auf die Konjunkturschwankungen der Industrieländer. Da die Rohstoffexporte und -preise zumindest zu einem großen Teil von der konjunkturellen Lage in den Industrieländern abhängen, werden sie in Boomzeiten direkt (durch Preissteigerungen) und indirekt (durch Mehrkäufe der Entwicklungsländer) inflationsverstärkend wirken, in Rezessionen werden sie hingegen den Abschwung noch verstärken (durch geringere Käufe der Dritten Welt). ${ }^{62}$ Eine Stabilisierung der Rohstoff preise würde also auch zu beträchtlichem Nutzen für die Konsumentenländer führen. Sie würde auch die Kalkulationsrisiken bei Rohstoffinvestitionen mindern helfen.Voraussetzung dieser positiven Effekte wären jedoch m. E. ausreichend groß ausgestattete Ausgleichslager. Hier sind wir an einem besonders kritischen Punkt angelangt: Von allen Autoren in der Bundesrepublik wird der vom UNCTAD-Sekretariat errechnete Finanzbedarf für den CF als zu niedrig eingeschätzt, teils weil Versicherungs-, Verpackungs-, und Frachtkosten der Rohstoffe nicht oder nicht adäquat berücksichtigt wurden, teils weil mit zu optimistischen Gewinnen der Rohstoffabkommen gerechnet werde, aber vor allem, weil der Umfang der Rohstofflager vom Sekretariat zu gering (auf 1,5 bis 3 Monatsraten der Rohstoffausfuhr) angesetzt sei. ${ }^{63}$ Es soll nicht untersucht werden, inwieweit diese Behauptungen zu Recht erhoben werden, hinweisen möchten wir aber auf die Gefahr, daß zu klein ausgelegte Ausgleichslager nur marginale Preisglättungen zuwege bringen (einer Simulation des Zinnmarktes zufolge, war das Zinnabkommen nur zu einer Dämpfung der jährlichen Preisfluktuationen von 10,4 auf 9,4\% in der Lage) ${ }^{64}$ und sich bald nach Etablierung des CF dann die Notwendigkeit paralleler erlösstabilisierender Maßnahmen o. ä. erweisen könnte. Gegen einen CF, der größer als bislang geplant ausfiele, müßte jedoch mit dem konzertierten Widerstand sämtlicher Ländergruppen gerechnet werden.

Als Fazit dieser Úberlegung wird man die Behauptung, der CF stelle ein Instrument globaler Planwirtschaft dar, als unbegründet, wenn nicht als durchsichtige Panikmache zurückweisen müssen. Diese Behauptung stützt sich vor allem auf die Annahme, der CF könne über

\footnotetext{
$60 \mathrm{Vgl} . \mathrm{TD} / \mathrm{B} / \mathrm{IPC} / \mathrm{CF} / 2, \mathrm{~S} .9$.

61 Interview bei der UNCTAD.

$62 \mathrm{Vgl}$. N. Kaldor, Inflation and Recession in the World Economy, in: The Economic Journal, Dec. 1976; K. P. Sauvant, The Poor Countries and the Rich - a Few Steps Forward, in: Dissent, Winter 1978, S. 43 ff.; C. F. Bergsten, Ein umfassendes Programm für internationale Rohstoffragen, in: Europa Archiv 20/1977, S. $711 \mathrm{ff}$.

63 M. Tietzel, a. a. O., S. 113 f.; O. Matzke, Anm. 12, S. 42 f.; D. Kebschull, Anm. 53, S. 105 f.; G. Florin, Maßnahmen zur Steuerung des Angebots, in: Dams/Groh, Anm. 53, S. 115; S. Baron/H. H. Glismann/B. Stecher, Internationale Rohstoff politik, Tübingen, 1977 , S. $15 \mathrm{ff}$.

64 Vgl. G. W. Smith/G.R. Schink, The International Tin Agreement: A Reassessment, in: The Economic Journal, Dec. 1976, S. 715 ff.
} 
dem Gleichgewichtspreis liegenden Rohstoffpreisen zum Durchbruch verhelfen. Dies ist aber schon deswegen unwahrscheinlich, weil ja den Preisen der Substitute Rechnung getragen werden muß. ${ }^{65}$ Wären langfristige Preiserhöhungen möglich, wäre ein Gemeinsamer Fonds überflüssig und Kartelle geeigneter. So gesehen ist das Begehren nach einem CF eher ein Zeichen für die schwache Position, in der sich Entwicklungsländer befinden.

\section{Der Common Fund - ein weiteres Entwicklungshilfeinstrument?}

Der CF ist seiner Idee nach ausdrücklich nicht als Entwicklungshilfeinstitution geplant, sondern soll den rohstoff produzierenden Ländern zu gleichsam selbstverdientem Einkommen verhelfen, nach einem kühnen Diktum einer UNCTAD-Publikation Entwicklungshilfe zu einem späteren Zeitpunkt gar überflüssig machen. ${ }^{66}$ Soll zumindest ersteres gewährleistet sein, so müssen die Gewinne aus den Rohstoffan- und -verkäufen ausreichen, um die Lagerhaltungskosten, die administrativen Kosten der ICAs und des CF und die Zinskosten für marktüblich Kredite aufzubringen. Die Industrieländer bestreiten nun, daß dies garantiert werden könne. In der Tat kommen selbst die etwas optimistischen Modellrechnungen des UNCTAD-Sekretariats nur auf Nettoprofite der ICAs in Höhe von 7,3 \% bzw. 7,6 \% p. a., die damit nicht ausreichen würden, um auch nur die Zinskosten für die bereits leicht vergünstigten Weltbankkredite $\left(8,85 \%\right.$ Zins p. a.) aufzubringen. ${ }^{67} \mathrm{Die}$,,Rentabilität" des $\mathrm{CF}$ im kommerziellen Sinne wird im wesentlichen nur durch die zinslosen Regierungsbeteiligungen (s. o.) und durch erhoffte günstige Kredite seitens internationaler Organisationen erreicht werden können. Diese Subventionen rücken den CF zumindest etwas in die Nähe der traditionellen Entwicklungshilfeinstrumente. Die Gegenargumentation der , $77^{\prime \prime}$ ist im wesentlichen moralischer Natur: ,,Stark vergünstigte Kredite des CF an die ICAs wären gerechtfertigt, da sie die ernsten Probleme lösen helfen würden, unter denen die Entwicklungsländer schon so lange leiden. $\operatorname{Er}$ (der Referent, jb) sei nicht der Ansicht, daß derlei Kredite das Konzept der Lebensfähigkeit einer Institution in Frage stellen würden. "68 Zum Entwicklungshilfecharakter des CF trägt weiterhin der von den , 77 “ ins Auge gefaßte hohe Anteil der Industrieländer an den Gesamtkosten bei, sollen sich doch die Kapitalsubskriptionen der Entwicklungsländer auf $18 \%$ der Gesamtsumme beschränken. Bei angenommener gleicher Nutzenverteilung für Produzenten und Konsumenten handelt es sich hier um einen indirekten Ressourcentransfer. Dazu kommt, daß die anderen Maßnahmen mit Mitteln finanziert werden sollen, deren Bedingungen denen der günstigsten Entwicklungshilfekredite angepaßt sein sollen. Dies wären immrerhin drei nicht unwesentliche Momente, die den geplanten CF in die Nähe eines weiteren Instruments indirekter Entwicklungshilfe rücken würden.

\section{Schwierige Rohstoff verhandlungen}

Den bisherigen Rohstoffabkommen (bei Weizen, Zucker, Kaffee, Kakao, Zinn und Olivenöl) werden meist nur geringe oder gar keine Erfolge bei der Erfüllung ihrer eigentlichen Aufgaben, nämlich der Stabilisierung der Preise und des Einhaltens der vereinbarten Preisgren-

65 D. Kebschull in Anm. 53, S. 100.

66 H. O'Neill, A Common Interest in a Common Fund, U. N., New York, 1977, S. 31 f.

67 Vgl. zu den Profiten: TD/B/IPC/CF/2, S. 19.

$68 \mathrm{Vgl}$. TD/B/IPC/CF/8, S. 11. 
zen attestiert. Abgesehen von der Fragwürdigkeit der vielfach verwendeten Erfolgsdefinition -immerhin wurde z. T. eine Preisstabilisierung von 5 bis $10 \%$ beobachtet - scheiterten die bisherigen Abkommen vor allem an Folgendem:

a. In fast allen Fällen traten wichtige Produzenten oder Konsumenten dem Abkommen nicht bei;

b. die Abkommen enthielten keinen automatischen Mechanismus, um die Preisbandbreiten dem Markttrend anzupassen, die nicht mehr adäquaten Preise bewirkten aber alsbald einen völligen Funktionsverlust der Abkommen (so v. a. bei Kaffee, Kakao und Zucker);

c. die Abkommen enthielten nur schwache oder keine Sanktionsmittel gegen Vertragsverletzungen wie etwa die Nichtbeachtung der Exportquoten;

d. die finanzielle Ausstattung und damit die Lagermengen der Abkommen waren meist zu gering;

e. schließlich waren von den bisherigen Abkommen im wesentlichen nur das Zinn- und das Kakaoabkommen in der Form (Bufferstock-Abkommen) realisiert worden, wie sie der Gemeinsame Fonds vorsieht, die anderen Abkommen funktionierten entweder nur als Exportquotenabkommen oder gar nur als Marktpflegeabkommen (Olivenöl). Das Zinnabkommen hinwieder wurde in seiner Wirksamkeit durch die strategischen Zinnvorräte der USA beeinträchtigt, da diese um ein Vielfaches höher lagen und An- und Verkäufe der beiden Lager nicht oder kaum miteinander abgestimmt wurden, während beim Kakaoabkommen der Marktpreis schon nach dessen Abschluß über der vertraglich vereinbarten Obergrenze lag, das Abkommen von Beginn an mangels Vorkehrungen zur Preisanpassung gar nicht funktionieren konnte. ${ }^{69}$

Die beschriebenen Mängel der bisherigen Abkommen können gleichzeitig als Anleitung besserer Vereinbarungen dienen, sie machen aber auch deutlich, daß der anders konzipierte CF nicht schon durch den Verweis auf die negativen Erfahrungen der Vergangenheit erledigt ist. Zwei Punkte sind dabei besonders von Belang:

1. Die bisher hauptsächlich in der Form von Exportquotenabkommen abgeschlossenen Rohstoffvereinbarungen bürden die Abkommenslasten (nämlich den Produktionsverzicht) einseitig den Produzenten auf und geben zu Vertragsverletzungen besonders leicht Anlaß (Produktion über die Quote hinaus bei Preisanstieg). Durch eine Einbeziehung aller Produzenten- und vor allem auch aller Konsumentenländer wäre eine präzisere Úberwachung der Exportmengen und eine Verhinderung von Käufen bei Abkommensaußenseitern möglich. Sodann lohnt sich ein Alleingang eines Produzenten eher bei individuell abgeschlossenen Rohstoffabkommen (Vorschlag der Gruppe B), als bei der Paketlösung des CF, wo sich Vor- und Nachteile aus verschiedenen Abkommen für die einzelnen Länder kompensieren mögen und wo die Kostenbeteiligung nicht direkt aus den Abkommen erfolgen soll (s. o.). Der CF in der von den , 77 “ vorgeschlagenen Form soll also auch - dies freilich selten eingestanden - unsolidarisches Handeln der Entwicklungsländer gegeneinander erschweren. ${ }^{70}$

2. Bufferstocks können nicht und sollen auch nicht dauerhaft gegen Markttrends auf steigende bzw. fallende Preise ankämpfen. Wegen ihrer zwangsläufig begrenzten Kapitalausstattung können sie nur eine relative Preisstabilisierung um den Markttrend herum

\footnotetext{
$69 \mathrm{Zu}$ den Erfolgen und Erfahrungen bisheriger Rohstoffabkommen: D. Kebschull et al., Anm. 12; M. Radetzki, International Commodity Market Agreements, London, 1970; H. Ch. Binswanger, Internationale Rohstoffabkommen - eine Darstellung, in: Außenwirtschaft, 1964, S. 350 ff.; D. Kebschull, Anm. 53; R. Senti, Monopolisierung im internationalen Rohwarenhandel, Diessenhofen, 1975; M. E. Streit, Einige alte Uberlegungen zu neuerlichen Schwierigkeiten in der internationalen Rohstoff politik, in: Konjunkturpolitik, 1976, S. 1 ff.; zum Kakaoabkommen: R. Hanisch, Kakaopolitik - Das Ringen der Entwicklungsländer mit den Industrieländern um die Regulierung des Rohstoffmarktes, in: Verfassung und Recht in Ubersee, 1/1978, S. 27 ff.; zum Kaffeeabkommen: B. S. Fisher, The International Coffee Agreement.

$70 \mathrm{Vgl}$. H. O’Neill, Anm. 66, S. 13.
} 
gewährleisten. Sollen bei der begrenzten Finanzmasse die künftigen Rohstoffabkommen nicht wie einige bisherige die meiste Zeit entweder durch überfüllte oder leere Lager funktionslos sein, sind periodische Anpassungen der Interventionspreise unausweichlich. Diese sollten, da die Einigung von Produzenten und Konsumenten hierüber so schwer ist, automatisch erfolgen, d. h. etwa an die Höhe der vorhandenen Lagervorräte gekoppelt, erfolgen.

Um nun auf die derzeitigen Realisierungschancen neuer Rohstoffabkommen innerhalb des CF zu kommen, so stehen diese denkbar schlecht: bislang zeichnet sich als einzig neues Abkommen eine Vereinbarung über Kautschuk ab, bei allen anderen Abkommen, sofern sie überhaupt zustande kommen, wird der in Nairobi zunächst gesetzte Termin (Ende 1978) weit überschritten werden, ja für manche Rohstoffe (Bananen und Bauxit) haben noch nicht einmal vorbereitende Treffen stattgefunden ${ }^{71}$. Gründe für diesen Mißerfolg liegen außer in den Verschleppungsstatistiken der Industrieländer (Anforderung weitere „Studien“, Einrichtung beratender Gremien usf.) auch in den viel berufenen Interessendivergenzen der Rohstoffländer selbst:

a) Ein Land mit einem hohen Anteil an den Exporten eines Rohstoffes ist damit faktisch Monopolist. Diese Stellung kann durch einen CF allenfalls geschmälert werden, der geradezu als Schutzschild der kleineren Anbieter figurieren könnte. Die größten Exporteure sind auch meist jene, die bei der Rohstoffproduktion am konkurrenzfähigsten sind, eine Situation, die durch den Schutz weniger konkurrenzfähiger Anbieter für sie nur beeinträchtigt würde.

b) Kostengünstig produzierende Rohstoffländer haben bei Abkommen ein Interesse an niedrigen Interventionspreisen, zu denen sie zwar ihre gesamte Produktion absetzen könnten, weniger konkurrenzfähige Anbieter aber vom Markt fegen würden, die daher an hohen Interventionspreisen interessiert sind.

c) Schwierig ist auch die Zuteilung von Exportquoten, da deren Orientierung am Exportzurückliegender Jahre das Aufkommen neuer Exporteure erschwert.

d) Ein Minimumpreis im Rahmen eines Abkommens schließt eine flexible Preispolitik etwa zur aggressiven Markteroberung oder zur Ausbootung von Konkurrenten aus; der Preis wäre also kein verkaufspolitisches Instrument mehr. Besonders aus diesem Grund laufen die in Entwicklungsländern ansässigen multinationalen Rohstoffkonzerne Sturm gegen den Beitritt ihrer Gastländer zu ICAs (in massiver Form geschieht dies v. a. seitens der Bananen- und Teekonzerne) ${ }^{\mathbf{7 2}}$.

Es erscheint nun allerdings wenig angebracht, wie dies leider immer wieder in der deutschen Diskussion geschieht, angesichts dieser Interessendivergenzen der Produzenten, in mehr oder minder lautes Triumphieren auszubrechen. Denn der CF, dies wohl nicht nur ein subjektiver Eindruck, wird auf jeden Fall realisiert werden. Dadurch, daß die Konsumentenländer den Abschluß einzelner Abkommen noch zusätzlich und massiv erschwert haben, zeichnet sich ein Resultat ab, das wohl niemanden befriedigen kann: die Etablierung eines Gemeinsamen Fonds als finanziellem Rahmen, der mit nichts (nämlich mit keinem ICA) ausgefüllt werden kann und funktionslos Kapital verschlingt.

$71 \mathrm{Vgl}$. TD/B/IPC/AC/L. 16 u. Add. 1.

72 Interview bei der UNCTAD. 


\section{Alternative Angebote der Industrieländer}

In der Bundesrepublik und teilweise auch in anderen westlichen Staaten herrschte lange Zeit der Glaube vor, durch ein Anerbieten ,,ordnungspolitisch unbedenklicher" Alternativen innerhalb und außerhalb des Rohstoffbereichs, die Länder der Dritten Welt von der Realisierung ,,weltweiten Staatshandels“ à la Common Fund abbringen zu können. Inhalte dieser, zuweilen unter reißerischen Etiketten (,,marktwirtschaftlich Gegenoffensive“) ${ }^{73}$ angebotenen Alternativen wären:

a) die Gewährung großzügiger Zollpräferenzen an Entwicklungsländer und allgemein eine verstärkte Handelsliberalisierung;

b) die Aufstockung der Entwicklungshilfe (nebst vermehrtem privatem Ressourcentransfer) sowie die Erleichterung der Schuldenlast der Entwicklungsländer;

c) die Erleichterung des Technologietransfers;

d) die vor allem von der Bundesregierung immer wieder ins Spiel gebrachte Exporterlösstabilisierung $\mathbf{7 4}^{\text {. }}$

Wie wir schon an anderer Stelle ausgeführt haben, ist die Realisierbarkeit dieser Alternativen außerordentlich gering ${ }^{75}$. Was heute zur Debatte steht ist - auch angesichts der Situation auf dem Arbeitsmarkt - nicht eine Beseitigung von Handelsschranken gegenüber Entwicklungsländern, sondern allenfalls eine Erhaltung des status quo ${ }^{76}$. Auch ist das Volumen der Entwicklungshilfe, real sowie bezogen auf das Bruttosozialprodukt der Geber, seit 1975 wieder stärker rückläufig und bei der Frage der Verschuldung wurden den Entwicklungsländern auf der Sitzung des Ministerrats der UNCTAD (März 1978) nur minimale Konzessionen gemacht: nicht nur, daß die Schulden an Private völlig ausgenommen wurden, es wurden auch nur die ärmsten Entwicklungsländer bedacht und dies auch nur derart, daß die Konditionen der heutigen Entwicklungshilfekredite an diese Länder rückwirkend auf die ihnen zu einem früheren Zeitpunkt gewährten ausgedehnt werden sollen. Dies außerdem nur als Empfehlung an die Geber ${ }^{77}$. Der erleichterte Technologietransfer meint im wesentlichen eine Aufstockung der Technischen Hilfe in diesem Bereich, Hilfe bei der Erstellung der staatlichen technologischen Infrastruktur in der Dritten Welt und Steuererleichterungen für in Entwicklungsländern sich niederlassende Betriebe, jedoch gerade nicht eine Verbesserung des privaten Patent- und Lizenzverkehrs zugunsten der Dritten Welt. Besonders kühn ist also die marktwirtschaftliche Offensive in praxi bislang nicht gewesen.

Es bleibt die Erporterlösstabilisierung, die vor allem in der Bundesrepublik lange (und zum Teil auch noch heute) als Alternative zum Rohstoff programm der , 77 “" gesehen worden ist. Diese soll die Exporterlöse direkt stabilisieren, indem in Höhe des Fehlbetrages zwischen aktuellen und prognostizierten Exporterlösen Kredite gewährt werden. Für dieses gleichsam weltweite STABEX-Modell als einen Gegenvorschlag zum CF konnte die Bundesregierung nicht einmal unter den Industrieländern Anhänger gewinnen. Die skandinavischen Staaten wären eventuell bereit gewesen, beide Vorhaben zu finanzieren, die anderen Industriestaaten, allen voran die USA, befürchten letztlich genau dazu gezwungen zu werden und winkten daher ab. Der Bundesregierung war diese ablehnende Haltung schon seit längerem bekannt, was zusammen mit der Tatsache, daß konkrete Modelle der Erlösstabilisierung in

73 Es erübrigt sich, Belege für die ,,marktkonformen“ Gegenvorschläge anzugeben, da hier eine ungeheure Fülle von statements in die nämliche Richtung zielen.

74 Ähnliche Vorschläge auch bei O. Matzke, Anm. 13.

75 Vgl. dazu J. Betz/V. Rittberger, Die Haltung der Bundesrepublik zu den Forderungen nach einer Neuen Weltwirtschaftsordnung und gegenüber institutionellen Ansätzen zu ihrer Verwirklichung, Papier für den Kongreß der DVPW 1977 in Bonn.

$76 \mathrm{Vgl}$. G. K. Helleiner, a. a. O.

77 Vgl. UNCTAD, Monthly Bulletin No. 139, April 1978. 
Bonn nie erarbeitet wurden, von ihrer Präsentation auf internationalen Konferenzen ganz zu schweigen, beinahe zwingend zu der Annahme führt, daß es sich beim deutschen Anerbieten der Erlösstabilisierung um einen Alibivorschlag handelte ${ }^{\mathbf{7 8}}$.

Die Beobachtung, daß die Entwicklungsländer an ihrem Konzept des CF festhalten, statt sich auf die marktkonformen Gegenvorschläge der Gruppe B einzulassen, ist also nicht nur auf ihre ,,Starrheit" und ihren ,Fanatismus" zurückzuführen, wie O. Matzke meint ${ }^{79}$, sondern auch auf die Dürftigkeit der Gegenangebote. Dazu kommt, daß eine Verstärkung der Entwicklungshilfe wie auch die Finanzierung exporterlösstabilisierender Maßnahmen von den Entwicklungsländern überwiegend als Maßnahmen der internationalen „,Sozialhilfe“ angesehen werden, die ihnen letztlich keine Einnahmen verschaffen, die vom Willen und der Laune der Industriestaaten unabhängig sind.

Die geringe Konzessionsbereitschaft der , 77 “ in der Frage des CF und seiner Modalitäten, wie auch noch stärker hinsichtlich möglicher Alternativen, rührt natürlich auch daher, daß der CF in der vorgeschlagenen Form eine Paketlösung darstellt, die jeder Entwicklungsländergruppe etwas gibt. Konzessionen aber würden manche Gruppen stärker treffen als andere und sind aher nur bedingt konsensfähig ${ }^{80}$.

Ubrigens lassen sich gegen das Modell der Exporterlösstabilisierung auch noch praktische und theoretische Argumente ins Feld führen, die seine Überlegenheit gegenüber dem CF zumindest in Frage zu stellen vermögen:

a) Bei der Erlösstabilisierung erfolgt ein Finanzausgleich ex post, also längere Zeit nach Eintritt der Einnahmeausfälle; bei der Preisstabilisierung, die ja auch die Einkommen der Produzenten stabilisieren soll, käme es idealiter erst gar nicht zu so großen Ausfällen;

b) die Finanzbedürfnisse eines weltweiten Erlösstabilisierungssystems wären vermutlich größer als jene des CF, da bei ersterem der ganze Exportausfall ausgeglichen wird, während ja beim CF bereits mit kleineren Summen Preis und Erlöse stabilisiert werden sollen;

c) die Erlösstabilisierung würde vor allem die in Konkurrenz zu Synthetika stehenden oder die unter einem Ubberangebot leidenden Rohstoffe ihren natürlichen Schrumpfprozessen überlassen und nicht diversifikationsfördernd eingreifen ${ }^{\mathbf{8 1}}$.

Ein gewichtiges Argument gegen preisstabilisierende Maßnahmen mittels Bufferstocks und für eine globale Erlösstabilisierung ist verstärkt in neuester Zeit wieder ins Feld geführt worden: preisstabilisierende Maßnahmen würden nämlich vor allem bei angebotsinduzierter Instabilität der Rohstoffpreise die Fluktuationen der Exporteinnahmen eher noch verstärken, also ihr eigentliches Ziel verfehlen! Bei Preissteigerungen aufgrund von Angebotsverknappungen (etwa bei einer schlechten Kaffeeernte in Brasilien) sinken ja in der Regel die nachgefragten Mengen, während bei Angebotserhöhungen die Preise sinken. Dieser MengenPreis-Effekt bewirkt eine quasi automatische Exporterlösstabilisierung, die bei einer Stabilisierung der Rohstoffpreise entfallen würde, wodurch Exporterlösfluktuationen tendenziell zunehmen $^{82}$.

Gar so durchschlagend ist freilich auch dieses Argument nicht. Einmal sind die empirischen Kenntnisse über die Ursachen von Rohstoffpreisschwankungen außerordentlich dürftig, zum anderen geht aus ihnen hervor, daß nur wenige, hauptsächlich agrarische Rohstoffe angebotsinduzierte Preisschwankungen aufweisen und zuletzt, und m. E. entscheidend, gilt

78 Dies wird durch Interviews im AA und im BMZ abgedeckt

79 O. Matzke, Anm. 26 an einigen Stellen.

80 Daher auch das ,,primitive Aufaddieren“ ihrer Forderungen (O. Matzke ebenda, S. 8).

81 Interview bei der UNCTAD.

82 Vgl. besonders: S. Baron, Preisstabilisierung als Instrument der Erlösstabilisierung - Eine modelltheoretische und empirische Kritik am ,Integrierten Rohstoffprogramm“, in: Die Weltwirtschaft, Heft 1, 1977, S. $175 \mathrm{ff}$

83 Vgl. D. Kebschull et al., Anm. 12; M. Radetzky, Anm. 69; S. Baron et al., Anm. 12. 
die Destabilisierung der Exporteinkünfte durch Preisstabilisierung nur auf globaler, nicht jedoch auf Länderebene. Wir wollen dies an einem zugestandenermaßen simplifizierten Beispiel demonstrieren, betonen daher, daß sich an der Aussage selbst durch realitätsnähere Zahlen nichts ändern würde.

\begin{tabular}{lrrrrrrrrrrrrrrr}
\hline & \multicolumn{1}{c}{1974} & \multicolumn{4}{c}{1975} & \multicolumn{4}{c}{ Preis } & \multicolumn{4}{c}{ Erlös } & \multicolumn{4}{c}{1976} \\
Land & \multicolumn{1}{c}{ Prod. Preis Erlös Prod. } & A & B & \multicolumn{1}{c}{ A } & B & Prod. & A & B & A & B B \\
\hline Bras. & 1000 & 1,0 & 1000 & 500 & 1,5 & 1,0 & 750 & 500 & 1300 & 0,75 & 1,0 & 975 & 1300 \\
Kol. & 200 & 1,0 & 200 & 100 & 1,5 & 1,0 & 150 & 100 & 300 & 0,75 & 1,0 & 225 & 300 \\
Kenia & 100 & 1,0 & 100 & 100 & 1,5 & 1,0 & 150 & 100 & 100 & 0,75 & 1,0 & 75 & 100 \\
Ghana & 100 & 1,0 & 100 & 100 & 1,5 & 1,0 & 150 & 100 & 100 & 0,75 & 1,0 & 75 & 100 \\
X & 100 & 1,0 & 100 & 100 & 1,5 & 1,0 & 150 & 100 & 100 & 0,75 & 1,0 & 75 & 100 \\
Y & 50 & 1,0 & 50 & 50 & 1,5 & 1,0 & 75 & 50 & 50 & 0,75 & 1,0 & 37,5 & 50 \\
Z & 50 & 1,0 & 50 & 50 & 1,5 & 1,0 & 75 & 50 & 50 & 0,75 & 1,0 & 37,5 & 50 \\
Global & 1600 & 1,0 & 1600 & 1000 & 1,5 & 1,0 & 1500 & 1000 & 2000 & 0,75 & 1,0 & 1500 & 2000 \\
\hline
\end{tabular}

$\mathrm{A}=$ ohne Preisstabilisierung $\quad \mathrm{B}=$ mit Preisstabilisierung

* Das Beispiel arbeitet mit der nicht unrealistischen Preiselastizität der Nachfrage von 0,8

Wie leicht zu ersehen, würde eine erfolgreiche Preisstabilisierung (bei einem Preis von 1,0) die Erlöse auf globaler Ebene destabilisieren (1600, 1000 und 2000 statt der nahezu stabilen Erlöse 1600, 1500 und 1600), nicht jedoch auf der Ebene aller einzelner Länder! Die Einkünfte der Länder, deren Angebot nicht oder nur geringfügig schwankt, würden stabilisiert. Dieser konstruierte Fall spiegelt insoweit die Realität, als nicht in allen rohstoff produzierenden Ländern gleichzeitig dieselben klimatischen Wechselfälle auftreten.

\section{Schluß}

Es ist mittlerweile zu einem Gemeinplatz kritisch angeleiteter Entwicklungsländerforschung geworden, in dem Programm der ,,Neuen Weltwirtschaftsordnung " nicht die Prinzipien einer versuchten strukturellen bzw. gar revolutionären Umgestaltung der Weltarbeitsteilung zu sehen, sondern Prinzipien, die sich im wesentlichen am überkommenen Leitbild des freien Welthandels orientieren (Senghaas), dessen Spielregeln zugunsten der Dritten Welt modifiziert werden sollen, um dadurch eine Erhöhung des Anteils der Entwicklungsländer am erwirtschafteten Mehrprodukt zu bewirken ${ }^{84}$. Die NWWO wird also bestenfalls als reformistisches Programm wahrgenommen, das die überkommene Weltarbeitsteilung verbessert und erträglicher gestaltet und damit womöglich die Aufrechterhaltung der Hegenomie der Zentren zu verlängern in der Lage ist $(\mathrm{Amin})^{\mathbf{8 5}}$. In dieser Perspektive gerät auch der CF zu einem Versuch, die Fixierung der armen Volkswirtschaften auf den Rohstoffsektor erträglicher zu gestalten ,,durch Verhinderung weiteren Kaufkraftverfalls der Dritten Welt und durch Reduktion der Exporterlösfluktuationen. Zudem . . . würde das IPC ein rationa-

$84 \mathrm{Vgl}$. D. Senghaas, Weltwirtschaftsordnung und Entwicklungspolitik, Frankfurt, 1977, S. 206 ff.; ähnlich S. Amin, Self-Reliance and the New International Economic Order, in: Monthly Review, July/August 1977.

85 Vgl. K. P. Sauvant, Anm. 62 u. S. Amin, a. a. O. 
leres Management der Rohstoffproduktion ermöglichen . . . Dadurch würden sowohl die Handhabung der Wirtschaftszyklen der Industrieländer erleichtert, wie auch die Planung des Entwicklungsprozesses der Länder der Dritten Welt" ${ }^{\text {‘86 }}$. Man ist versucht zu sagen: Immerhin! Offenbar aber gibt es nur wenige denkbare Reformen der Weltwirtschaftsordnung, die vor den kritischen Augen mancher Theoretiker Gnade finden könnten. Gerechterweise dürfte man allerdings Reformvorhaben, die sich nicht an der Strategie der ,, autozentrierten Entwicklung" ausrichten - so sehr man dies auch bedauern mag - auch nicht an deren Maßstäben messen, zumal mit dieser Strategie ohnedies kein Vorhaben vereinbar wäre (wie der $\mathrm{CF}$ ), das lediglich auf eine Verbesserung der Arbeitsteilung zwischen Industrie- und Entwicklungsländern hinausläuft und/oder die unterstützende Mitwirkung der reichen Staaten zur Voraussetzung hätte. Verständlich ist die Tendenz, die NWWO und darin den CF mit dem Etikett ,,liberal“ oder ,,reformistisch“ zu versehen als Reaktion auf andere, viel zu hoch gehängte Befürchtungen oder Erwartungen, die den CF entweder als superdirigistisches Machwerk verteufelt oder als Kernstück einer antimonopolistischen Weltwirtschaftsordnung feiern.

Ein ähnlicher, und bei Vergleichsfällen sicher zu Recht hervorgehobener Gesichtspunkt, wäre die Frage, ob mit den für den CF aufgewandten Mitteln nicht sinnvoller die Grundbedürfnisse breiter Massen in Entwicklungsländern befriedigt werden sollten. In der Tat ist ja keine Gewähr dafür gegeben, daß die Nutzen aus der Tätigkeit des Fonds, die zunächst bei den in der Rohstoffextraktion Beschäftigten (Rohstoffkonzerne, Plantagen, Kleinbetriebe, Arbeiter) und den Regierungen anfallen (als gleichmäßige Steuereinnahmen), irgendwann einmal zu breiteren Schichten außerhalb des Rohstoffsektors durchsickern. Nun ist aber $\mathrm{m}$. E. die Gegenüberstellung: Finanzierung des CF versus Finanzierung von Projekten zur Befriedigung der Grundbedürfnisse keine echte Alternative. Denn abgesehen davon, daß die Spielräume für derartige Leistungen der Entwicklungshilfe äußerst bescheiden sind ${ }^{87}$, würde auch eine Befriedigung der Grundbedürfnisse ohne einen komplementären Abbau der Handelshemmnisse, eine Verbesserung der Produktionsstrukturen, eine Erleichterung des Technologietransfers und auch ohne eine Stabilisierung der Rohstoffpreise auf wenig mehr als eine ,,globale Wohltätigkeitsaktion"

Soll die Idee des CF an ihren eigenen Zielsetzungen gemessen werden - also etwa an den von Sauvant (Anm. 86) genannten Funktionen eines CF - so ergab unsere Analyse eine Reihe möglicher Erfolgsbedingungen eines Gemeinsamen Fonds, die Beachtung verdienen:

a) Optimierung und nicht Minimierung der Kapitalausstattung;

b) großzügige Finanzierung der anderen Maßnahmen insbesondere bei Projekten, die die lokale Rohstoffverarbeitung fördern und Ủberproduktionen abbauen helfen;

c) keine Marktinterventionen des CF außerhalb von Rohstoffabkommen, damit eine Gefährdung der Kapitalausstattung vermieden wird;

d) strikte interne Höchstlimits der CF-Kredite an die ICAs;

e) Einzahlung der Kapitalsubskriptionen in den CF nach Maßgabe des Abschlusses von Rohstoffabkommen und in Höhe der proportional hierfür eingeplanten Finanzvolumina;

f) periodische, möglichst automatische Anpassung der Interventionspreise an die Marktlage sowie periodische Neufestsetzungen von Exportquoten und Rohstofflagermengen.

Es wird in der nächsten Zeit darauf ankommen adäquate Vorkehrungen für ein möglichst optimales Funktionieren des Gemeinsamen Fonds zu treffen und nicht darauf, den Entwick-

86 K. P. Sauvant, a. a. O., S. 47

87 Vgl. J. Betz, Die Internationalisierung der Entwicklungshilfe, Baden-Baden, 1978.

88 So treffend Paul P. Streeten, Ein Zielkonflikt? in: Forum Vereinte Nationen, Juni/Juli 1978. 
lungsländern noch möglichst viel ihrer Vorstellungen abzuhandeln, gleichgültig, ob diese sinnvoll oder weniger sinnvoll sind. Sonst wird zuletzt ein CF etabliert, der etwa aufgrund zu geringer Kapitalausstattung oder vager interner Verfahrensregeln jene Probleme, zu deren Lösung er konzipiert wurde, nicht lösen helfen kann und dann mit Sicherheit bald von neuerlichen Forderungen im Rohstoffbereich abgelöst wird.

\section{Nachtrag}

Nach schwierigen Konsultationen über die Wiederaufnahme der Verhandlungskonferenz über einen Gemeinsamen Fonds zeichnete sich dort bereits im November 1978 die Möglichkeit eines Kompromisses ab, der bei der Verhandlungsrunde im März 1979 eine Einigung über die grundsätzlichen Elemente des CF folgte. Im Gegensatz zu Presseberichten, die dieses Ergebnis vor allem der Konzessionsfreudigkeit der Industrieländer zurückführten, muß betont werden, daß der erzielte Kompromiß zumindest den hauptsächlichen Bedenken dieser Staaten in starkem Maße trägt. Das erste Fenster des CF (zur Preisstabilisierung) wird nunmehr mit lediglich 400 Mill. Dollar Kapital ausgestattet - wovon 150 Mill. an Regierungsbeiträgen in bar eingezahlt werden - die ausschließlich zur Bestreitung der administrativen Kosten und als Reservefonds für Liquiditätsengpässe dienen sollen (Inverventionen außerhalt ICAa und Subventionierung dieser ICAsentfallen damit). Wichtiger ist, daß die Rohstoffabkommen selbst nun die Hauptbausteine des CF sein werden und 1/3 ihrer maximalen Finanzbedürfnisse selbst aufbringen müssen.

Das zweite Fenster ist ebenfalls bezüglich seines Volumens und seiner Aufgabenstellung drastisch reduziert. Seine Kapitalausstattung soll 350 Mill. Dollar betragen, von denen 280 Mill. aus freiwilligen Beiträgen gespeist werden sollen, seine Aufgaben sollen sich auf Forschung und Entwicklung, Produktivitätsverbesserung und technische Hilfe für die vertikale Diversifikation beschränken.(a)

Dieser Kompromiß, der noch durch Detailregelung zu konkretisiren ist, vermag m. E. das globale Stabilisierungs-, Investitions- und Diversifikationsproblem bei Rohstoffen nicht zu lösen. Es ist schwer zu sehen, wie bei der hohen Eigenkapitalanforderungen an die ICAs und den entsprechend hohen Belastungen für die Mitgliedsländer, sich viele und gerade die kreditwürdisten Rohstoffabkommen zur Teilnahme am CF bereitfinden sollen (sie können auch den Kapitalmarkt in Anspruch nehmen), zumal die Chancen für den Abschluß neuer Rohstoffabkommen ohnedies schlecht sind.

Es ist auch schwer zu sehen, wie das zweite Fenster mit seiner mageren Finanzausstattung und entsprechend geringen Einflußchancen, die Rohstoffaktivitäten der anderen internationalen Finanzinstitutionen beeinflussen soll. Vielmehr droht nunmehr ein ungeplantes Wuchern von Projektinitiativen einer um ein zweites Fenster vermehrten Organisationenvielfalt.

(a) vgl. UNCTAD/CA/1329 GE. 79-51553; UNCTAD Presse Release, TAD/INF/1038, 20 March 1979; NZZ v. 22. 3. 79, Durchbruch beim Gemeinsamen Fonds? 


\title{
A Claim to Foreign Aid?
}

\author{
By Albert BleckmanN
}

The article discusses potential bases for claims of developing countries to foreign aid under international law. The current aid-practice cannot by itself create such a claim, but it is not completely irrelevant. It might become relevant under the principle of estoppel. The Charter of Economic Rights and Duties of States of 1974 only has the character of "soft law". As such, it might be a factor in establishing "abus de droit". Within this framework, the article submits that resolutions of the UN, doctrine, practice, comparative law etc. as a whole might be used to form the legal opinion of an international judge. Therefore it does not matter that state practice or comparative law alone cannot constitute a rule of law. In addition the article discusses whether the "Sozialstaatsprinzip" can be found in international law and whether a claim to foreign aid can be derived from the injustices of colonialism or the present economic order or from international human rights. As a result, the author states that while today there is no claim under international law to a specific amount of foreign aid, legal development points in this direction, and the refusal of any foreign aid at all is already a violation of international law.

\section{The Common Fund}

\section{BY JOACHIM BETZ}

The political attitude of the major industrial countries towards the demands of the Group of 77 for a Common Fund changed considerably in course of time. Critisizing the Fund initially as an instrument for the bureaucratic reshaping of the world commodity trade, these countries accepted at last the idea of price stabilization for raw materials and put forward on their part proposals for the setting up of a Common Fund.

This Fund has in fact never been, according to its conception, an attempt at global planning but an attempt at stabilizing commodity prices along the long-term market trend and to improve the bargaining position of the commodity exporting developing countries. On the other hand, the Common Fund (as proposed by the Group of 77) is not an instrument totally devoid of aid elements as it contains some scarcely disguised subsidies for the financing of stocking activities and other measures. The readiness of the industrial countries to accept finally the financing of price stabilization measures on a much broader scale than they were prepared to hitherto, is due to their changed perceptions of the possible merits and dangers of a Common Fund and, above all, to the fact that they are inable to make concessions in other important fields (trade, technology and resource transfer) of the North-South dialogue. It will be important in the course of the ongoing negotiations on the Common Fund and on new commodity aggreements - especially in consideration of the already considerably diverging interests of the producing countries themselves - to optimize and not to mimimize their results. Otherwise we will see the establishment of just another impotent mini-fund which will hardly be able to tackle the global commodity problems. 